\title{
Time-Dependent Toroidal Compactification Proposals and the Bianchi Type I Model: Classical and Quantum Solutions
}

\author{
L. Toledo Sesma, J. Socorro, and O. Loaiza \\ Departamento de Física, DCI, Universidad de Guanajuato, Campus León, Loma del Bosque No. 103 Colonia Lomas del Campestre, \\ Apartado Postal E-143, 37150 León, GTO, Mexico
}

Correspondence should be addressed to J. Socorro; socorro@fisica.ugto.mx

Received 19 January 2016; Revised 22 April 2016; Accepted 5 May 2016

Academic Editor: Elias C. Vagenas

Copyright (C) 2016 L. Toledo Sesma et al. This is an open access article distributed under the Creative Commons Attribution License, which permits unrestricted use, distribution, and reproduction in any medium, provided the original work is properly cited. The publication of this article was funded by SCOAP S $^{3}$

\begin{abstract}
We construct an effective four-dimensional model by compactifying a ten-dimensional theory of gravity coupled with a real scalar dilaton field on a time-dependent torus. This approach is applied to anisotropic cosmological Bianchi type I model for which we study the classical coupling of the anisotropic scale factors with the two real scalar moduli produced by the compactification process. Under this approach, we present an isotropization mechanism for the Bianchi I cosmological model through the analysis of the ratio between the anisotropic parameters and the volume of the Universe which in general keeps constant or runs into zero for late times. We also find that the presence of extra dimensions in this model can accelerate the isotropization process depending on the momenta moduli values. Finally, we present some solutions to the corresponding Wheeler-DeWitt (WDW) equation in the context of standard quantum cosmology.
\end{abstract}

\section{Introduction}

The 2015 release of Planck data has provided a detailed map of cosmic microwave background (CMB) temperature and polarization allowing us to detect deviations from an isotropic early Universe [1]. The evidence given by these data leads us to the possibility of considering that there is no exact isotropy, since there exist small anisotropy deviations of the $\mathrm{CMB}$ radiation and apparent large angle anomalies. In that context, there have been recent attempts to fix constraints on such deviations by using the Bianchi anisotropic models [2]. The basic idea behind these models is to consider the present observational anisotropies and anomalies as imprints of an early anisotropic phase on the CMB which in turn can be explained by the use of different Bianchi models. In particular, Bianchi I model seems to be related to large angle anomalies [2] (and references therein).

In a different context, attempts to understand diverse aspects of cosmology, as the presence of stable vacua and inflationary conditions, in the framework of supergravity and string theory have been considered in the last years [3-10]. One of the most interesting features emerging from these types of models consists in the study of the consequences of higher dimensional degrees of freedom on the cosmology derived from four-dimensional effective theories [11, 12]. The usual procedure for that is to consider compactification on generalized manifolds, on which internal fluxes have back-reacted, altering the smooth Calabi-Yau geometry and stabilizing all moduli [10].

The goal of the present work is to consider in a simple model some of the above two perspectives; that is, we will consider the presence of extra dimensions in a Bianchi I model with the purpose of tracking down the influence of moduli fields in its isotropization. For that we will consider an alternative procedure concerning the role played by moduli. In particular we will not consider the presence of fluxes, as in string theory, in order to obtain a moduli-dependent scalar potential in the effective theory. Rather, we are going to promote some of the moduli to time-dependent fields by considering the particular case of a ten-dimensional gravity coupled to a time-dependent dilaton compactified on a 6dimensional torus with a time-dependent Kähler modulus. With the purpose to study the influence of such fields, we are going to ignore the dynamics of the complex structure 
modulos (for instance, by assuming that it is already stabilized by the presence of a string field in higher scales). This will allow us to construct classical effective models with two moduli.

However, we are also interested in possible quantum aspects of our model. Quantum implications on cosmology from more fundamental theories are expected due to different observations. For instance, it has been pointed out that the presence of extra dimensions leads to an interesting connection to the ekpyrotic model [13], which generated considerable activity $[8,9,14]$. The essential ingredient in these models (see, for instance, [11]) is to consider an effective action with a graviton and a massless scalar field, the dilaton, describing the evolution of the Universe, while incorporating some of the ideas of pre-big-bang proposal $[15,16]$ in that the evolution of the Universe began in the far past. On the other hand, it is well known that relativistic theories of gravity such as general relativity or string theories are invariant under reparametrization of time. Quantization of such theories presents a number of problems of principle known as "the problem of time" $[17,18]$. This problem is present in all systems whose classical version is invariant under time reparametrization, leading to its absence at the quantum level. Therefore, the formal question involves how to handle the classical Hamiltonian constraint, $\mathscr{H} \approx 0$, in the quantum theory. Also, connected with the problem of time is the "Hilbert space problem" $[17,18]$ referring to the not at all obvious selection of the inner product of states in quantum gravity and whether there is a need for such a structure at all. The above features, as it is well known, point out the necessity to construct a consistent theory of gravity at quantum level.

Analyses of effective four-dimensional cosmologies derived from $\mathrm{M}$-theory and Type IIA string theory were considered in [19-21] where string fluxes are related to the dynamical behavior of the solutions. A quantum description of the model was studied in $[22,23]$ where a flat FriedmannRobertson-Walker geometry was considered for the extended four-dimensional space-time, while a geometry given by $S^{1} \times$ $T^{6}$ was assumed for the internal seven-dimensional space; however, the dynamical fields are in the quintom scheme, since one of the fields has a negative energy. Under a similar perspective, we study the Hilbert space of quantum states on a Bianchi I geometry with two time-dependent scalar moduli derived from a ten-dimensional effective action containing the dilaton and the Kähler parameter from a six-dimensional torus compactification. We find that, in this case, the wave function of this Universe is represented by two factors, one depending on moduli and the second one depending on gravitational fields. This behavior is a property of all cosmological Bianchi Class A models.

The work is organized in the following form. In Section 2 we present the construction of our effective action by compactification on a time-dependent torus, while in Section 3 we study its Lagrangian and Hamiltonian descriptions using as toy model the Bianchi type I cosmological model. Section 4 is devoted to finding the corresponding classical solutions for few different cases involving the presence or absence of matter content and the cosmological constant term. Using the classical solutions found in previous sections, we present an isotropization mechanism for the Bianchi I cosmological model, through the analysis of the ratio between the anisotropic parameters and the volume of the Universe, showing that, in all the cases we have studied, its value keeps constant or runs into zero for late times. In Section 5 we present some solutions to the corresponding WheelerDeWitt (WDW) equation in the context of standard quantum cosmology and finally our conclusions are presented in Section 6.

\section{Effective Model}

We start from a ten-dimensional action coupled with a dilaton (which is the bosonic component common to all superstring theories), which after dimensional reduction can be interpreted as a Brans-Dicke like theory [24]. In the string frame, the effective action depends on two spacetimedependent scalar fields: the dilaton $\Phi\left(x^{\mu}\right)$ and the Kähler modulus $\sigma\left(x^{\mu}\right)$. For simplicity, in this work we will assume that these fields only depend on time. The high-dimensional (effective) theory is therefore given by

$$
\begin{aligned}
S= & \frac{1}{2 \kappa_{10}^{2}} \int d^{10} X \sqrt{-\widehat{G}} e^{-2 \Phi}\left[\widehat{\mathscr{R}}^{(10)}+4 \widehat{G}^{M N} \nabla_{M} \Phi \nabla_{N} \Phi\right] \\
& +\int d^{10} X \sqrt{-\widehat{G} \widehat{\mathscr{L}}_{m}},
\end{aligned}
$$

where all quantities $\widehat{q}$ refer to the string frame while the tendimensional metric is described by

$$
d s^{2}=\widehat{G}_{M N} d X^{M} d X^{N}=\widehat{g}_{\mu \nu} d x^{\mu} d x^{\nu}+h_{m n} d y^{m} d y^{n}
$$

where $M, N, \ldots$ are the indices of the ten-dimensional space and Greek indices $\mu, v, \ldots=0, \ldots, 3$ and Latin indices $m, n, \ldots=4, \ldots, 9$ correspond to the external and internal spaces, respectively. We will assume that the six-dimensional internal space has the form of a torus with a metric given by

$$
h_{m n}=e^{-2 \sigma(t)} \delta_{m n}
$$

with $\sigma$ being a real parameter.

Dimensionally reducing the first term in (1) to fourdimensions in the Einstein frame (see the Appendix for details) gives

$$
\begin{gathered}
S_{4}=\frac{1}{2 \kappa_{4}^{2}} \int d^{4} x \sqrt{-g}\left(\mathscr{R}-2 g^{\mu \nu} \nabla_{\mu} \phi \nabla_{\nu} \phi\right. \\
\left.-96 g^{\mu \nu} \nabla_{\mu} \sigma \nabla_{\nu} \sigma-36 g^{\mu \nu} \nabla_{\mu} \phi \nabla_{\nu} \sigma\right),
\end{gathered}
$$

where $\phi=\Phi+(1 / 2) \ln (\widehat{V})$, with $\widehat{V}$ given by

$$
\widehat{V}=e^{6 \sigma(t)} \operatorname{Vol}\left(X_{6}\right)=\int d^{6} y
$$

By considering only a time-dependence on the moduli, one can notice that, for the internal volume $\operatorname{Vol}\left(X_{6}\right)$ to be small, the modulus $\sigma(t)$ should be a monotonic increasing function 
on time (recall that $\sigma$ is a real parameter), while $\widehat{V}$ is time and moduli independent.

Now, concerning the second term in (1), we will require properly defining the ten-dimensional stress-energy tensor $\widehat{T}_{M N}$. In the string frame it takes the form

$$
\widehat{T}_{M N}=\left(\begin{array}{cc}
\widehat{T}_{\mu \nu} & 0 \\
0 & \widehat{T}_{m n}
\end{array}\right),
$$

where $\widehat{T}_{\mu \nu}$ and $\widehat{T}_{m n}$ denote the four- and six-dimensional components of $\widehat{T}_{M N}$. Observe that we are not considering mixing components of $\widehat{T}_{M N}$ among internal and external components although nonconstrained expressions for $\widehat{T}_{M N}$ have been considered in [25]. In the Einstein frame the fourdimensional components are given by

$$
T_{\mu \nu}=e^{2 \phi} \widehat{T}_{\mu \nu}
$$

It is important to remark that there are some dilemmas about what is the best frame to describe the gravitational theory. Here in this work we have taken the Einstein frame. Useful references to find a discussion about string and Einstein frames and its relationship in the cosmological context are [26-30].

Now with expression (4) we proceed to build the Lagrangian and the Hamiltonian of the theory at the classical regime employing the anisotropic cosmological Bianchi type I model. The moduli fields will satisfy the Klein-Gordon like equation in the Einstein frame as an effective theory.

\section{Classical Hamiltonian}

In order to construct the classical Hamiltonian, we are going to assume that the background of the extended space is described by a cosmological Bianchi type I model. For that, let us recall here the canonical formulation in the ADM formalism of the diagonal Bianchi Class A cosmological models; the metric has the form

$$
d s^{2}=-N(t) d t^{2}+e^{2 \Omega(t)}\left(e^{2 \beta(t)}\right)_{i j} \omega^{i} \omega^{j}
$$

where $N(t)$ is the lapse function, $\beta_{i j}(t)$ is a $3 \times 3$ diagonal matrix, $\beta_{i j}=\operatorname{diag}\left(\beta_{+}+\sqrt{3} \beta_{-}, \beta_{+}-\sqrt{3} \beta_{-},-2 \beta_{+}\right), \Omega(t)$ and $\beta_{ \pm}$ are scalar functions, known as Misner variables, and $\omega^{i}$ are one-forms that characterize each cosmological Bianchi type model [31] and obey the form $d \omega^{i}=(1 / 2) C_{j k}^{i} \omega^{j} \wedge \omega^{k}$, with $C_{j k}^{i}$ the structure constants of the corresponding model. The one-forms for the Bianchi type I model are $\omega^{1}=d x, \omega^{2}=d y$, and $\omega^{3}=d z$. So, the corresponding metric of the Bianchi type I in Misner's parametrization has the form

$$
d s_{I}^{2}=-N^{2} d t^{2}+R_{1}^{2} d x^{2}+R_{2}^{2} d y^{2}+R_{3}^{2} d z^{2}
$$

where $\left\{R_{i}\right\}_{i=1}^{3}$ are the anisotropic radii and they are given by

$$
\begin{aligned}
& R_{1}=e^{\Omega+\beta_{+}+\sqrt{3} \beta_{-},} \\
& R_{2}=e^{\Omega+\beta_{+}-\sqrt{3} \beta_{-}}, \\
& R_{3}=e^{\Omega-2 \beta_{+}}, \\
& V=R_{1} R_{2} R_{3}=e^{3 \Omega},
\end{aligned}
$$

where $V$ is the volume function of this model.

The Lagrangian density, with a matter content given by a barotropic perfect fluid and a cosmological term, has a structure, corresponding to an energy-momentum tensor of perfect fluid [32-36]

$$
T_{\mu \nu}=(p+\rho) u_{\mu} u_{\nu}+p g_{\mu \nu}
$$

that satisfies the conservation law $\nabla_{\nu} T^{\mu \nu}=0$. Taking the equation of state $p=\gamma \rho$ between the energy density and the pressure of the comovil fluid, a solution is given by $\rho=$ $C_{\gamma} e^{-3(1+\gamma) \Omega}$ with $C_{\gamma}$ the corresponding constant for different values of $\gamma$ related to the Universe evolution stage. Then, the Lagrangian density reads

$$
\begin{aligned}
\mathscr{L}_{\text {matt }} & =16 \pi G_{N} \sqrt{-g} \rho+2 \sqrt{-g} \Lambda \\
& =16 N \pi G_{N} C_{\gamma} e^{-3(1+\gamma) \Omega}+2 N \Lambda e^{3 \Omega}
\end{aligned}
$$

while the Lagrangian that describes the fields dynamics is given by

$$
\begin{aligned}
\mathscr{L}_{I} & =\frac{e^{3 \Omega}}{N}\left[6 \dot{\Omega}^{2}-6 \dot{\beta}_{+}^{2}-6 \dot{\beta}_{-}^{2}+96 \dot{\sigma}^{2}+36 \dot{\phi} \dot{\sigma}+2 \dot{\phi}^{2}\right. \\
& \left.+16 \pi G N^{2} \rho+2 \Lambda N^{2}\right]
\end{aligned}
$$

using the standard definition of the momenta, $\Pi_{q^{\mu}}=$ $\partial \mathscr{L} / \partial \dot{q}^{\mu}$, where $q^{\mu}=\left(\Omega, \beta_{+}, \beta_{-}, \phi, \sigma\right)$, and we obtain

$$
\begin{aligned}
& \Pi_{\Omega}=\frac{12 e^{3 \Omega} \dot{\Omega}}{N}, \quad \dot{\Omega}=\frac{N e^{-3 \Omega} \Pi_{\Omega}}{12}, \\
& \Pi_{ \pm}=-\frac{12 e^{3 \Omega} \dot{\beta}_{ \pm}}{N}, \quad \dot{\beta}_{ \pm}=-\frac{N e^{-3 \Omega} \Pi_{\beta_{ \pm}}}{12}, \\
& \Pi_{\phi}=\frac{e^{3 \Omega}}{N}[36 \dot{\sigma}+4 \dot{\phi}], \quad \dot{\phi}=\frac{N e^{-3 \Omega}}{44}\left[3 \Pi_{\sigma}-16 \Pi_{\phi}\right], \\
& \Pi_{\sigma}=\frac{e^{3 \Omega}}{N}[192 \dot{\sigma}+36 \dot{\phi}], \quad \dot{\sigma}=\frac{N e^{-3 \Omega}}{132}\left[9 \Pi_{\phi}-\Pi_{\sigma}\right],
\end{aligned}
$$

and, introducing them into the Lagrangian density, we obtain the canonical Lagrangian as $\mathscr{L}_{\text {canonical }}=\Pi_{q^{\mu}} \dot{q}^{\mu}-N \mathscr{H}$. When we perform the variation of this canonical lagrangian with respect to $N, \delta \mathscr{L}_{\text {canonical }} / \delta N=0$, implying the constraint $\mathscr{H}_{I}=0$. In our model the only constraint corresponds to 
Hamiltonian density, which is weakly zero. So, we obtain the Hamiltonian density for this model:

$$
\begin{gathered}
\mathscr{H}_{I}=\frac{e^{-3 \Omega}}{24}\left[\Pi_{\Omega}^{2}-\Pi_{+}^{2}-\Pi_{-}^{2}-\frac{48}{11} \Pi_{\phi}^{2}+\frac{18}{11} \Pi_{\phi} \Pi_{\sigma}\right. \\
\left.-\frac{1}{11} \Pi_{\sigma}^{2}-384 \pi G_{N} \rho_{\gamma} e^{3(1-\gamma) \Omega}-48 \Lambda e^{6 \Omega}\right] .
\end{gathered}
$$

We introduce a new set of variables in the gravitational part given by $e^{\beta_{1}+\beta_{2}+\beta_{3}}=e^{3 \Omega}=V$ which corresponds to the volume of the Bianchi type I Universe, in similar way to the flat Friedmann-Robetson-Walker metric (FRW) with a scale factor. This new set of variables depends on Misner variables as

$$
\begin{aligned}
& \beta_{1}=\Omega+\beta_{+}+\sqrt{3} \beta_{-}, \\
& \beta_{2}=\Omega+\beta_{+}-\sqrt{3} \beta_{-}, \\
& \beta_{3}=\Omega-2 \beta_{+},
\end{aligned}
$$

from which the Lagrangian density (13) can be transformed as

$$
\begin{aligned}
\mathscr{L}_{I} & =\frac{e^{\beta_{1}+\beta_{2}+\beta_{3}}}{N}\left(2 \dot{\phi}^{2}+36 \dot{\phi} \dot{\sigma}+96 \dot{\sigma}^{2}+2 \dot{\beta}_{1} \dot{\beta}_{2}\right. \\
& +2 \dot{\beta}_{1} \dot{\beta}_{3}+2 \dot{\beta}_{3} \dot{\beta}_{2}+2 \Lambda N^{2} \\
& \left.+16 \pi G N^{2} \rho_{\gamma} e^{-(1+\gamma)\left(\beta_{1}+\beta_{2}+\beta_{3}\right)}\right),
\end{aligned}
$$

and the Hamiltonian density reads

$$
\begin{aligned}
\mathscr{H}_{I} & =\frac{1}{8} e^{-\left(\beta_{1}+\beta_{2}+\beta_{3}\right)}\left[-\Pi_{1}^{2}-\Pi_{2}^{2}-\Pi_{3}^{2}+2 \Pi_{1} \Pi_{2}\right. \\
& +2 \Pi_{1} \Pi_{3}+2 \Pi_{2} \Pi_{3}-\frac{16}{11} \Pi_{\phi}^{2}+\frac{6}{11} \Pi_{\phi} \Pi_{\sigma}-\frac{1}{33} \Pi_{\sigma}^{2} \\
& \left.-128 \pi G \rho_{\gamma} e^{(1-\gamma)\left(\beta_{1}+\beta_{2}+\beta_{3}\right)}-16 \Lambda e^{2\left(\beta_{1}+\beta_{2}+\beta_{3}\right)}\right] .
\end{aligned}
$$

So far, we have built the classical Hamiltonian density from a higher dimensional theory; this Hamiltonian contains a barotropic perfect fluid, that we have added explicitly. The next step is to analyze three different cases involving terms in the classical Hamiltonian (18) and find solutions to each of them in the classical regime.

3.1. Isotropization. The current observations of the cosmic background radiation set a very stringent limit to the anisotropy of the Universe [37]; therefore, it is important to consider the anisotropy of the solutions. We will denote through all our study derivatives of functions $F$ with respect to $\tau=N t$ by $F^{\prime}$ (the following analysis is similar to this presented recently in the K-essence theory, since the corresponding action is similar to this approach [38]). Recalling the Friedmann like equation to the Hamiltonian density

$$
\begin{aligned}
\Omega^{\prime 2}= & \beta_{+}^{\prime 2}+\beta_{-}^{\prime 2}+16 \sigma^{\prime 2}+\frac{1}{3} \phi^{\prime 2}+6 \phi^{\prime} \sigma^{\prime} \\
& +\frac{8}{3} \pi G \mu_{\gamma} e^{-3(1+\gamma) \Omega}+\frac{\Lambda}{3},
\end{aligned}
$$

we can see that isotropization is achieved when the terms with $\beta_{ \pm}^{\prime 2}$ go to zero or are negligible with respect to the other terms in the differential equation. We find in the literature the criteria for isotropization, among others, $\left(\beta_{+}^{\prime 2}+\beta_{-}^{\prime 2}\right) / H^{2} \rightarrow 0$, $\left(\beta_{+}^{\prime 2}+\beta_{-}^{\prime 2}\right) / \rho \rightarrow 0$, that are consistent with our above remark. In the present case the comparison with the density should include the contribution of the scalar field. We define an anisotropic density $\rho_{a}$ that is proportional to the shear scalar,

$$
\rho_{a}=\beta_{+}^{\prime 2}+\beta_{-}^{\prime 2},
$$

and will compare it with $\rho_{\gamma}, \rho_{\phi}$, and $\Omega^{\prime 2}$. From the Hamilton analysis we now see that

$$
\begin{aligned}
& \dot{\Pi}_{\phi}=0 \longrightarrow \Pi_{\phi}=p_{\phi}=\text { constant, } \\
& \dot{\Pi}_{\sigma}=0 \longrightarrow \Pi_{\sigma}=p_{\sigma}=\text { constant, } \\
& \dot{\Pi}_{+}=0 \longrightarrow \Pi_{+}=p_{+}=\text {constant, } \\
& \dot{\Pi}_{-}=0 \longrightarrow \Pi_{-}=p_{-}=\text {constant, }
\end{aligned}
$$

and the kinetic energy for the scalars fields $\phi$ and $\sigma$ are proportional to $e^{-6 \Omega}$. This can be seen from definitions of momenta associated with Lagrangian (13) and the above equations. Then, defining $\kappa_{\Omega}$ as $\kappa_{\Omega}^{2} \sim p_{+}^{2}+p_{-}^{2}+\left(16 / 132^{2}\right)\left(p_{\sigma}-\right.$ $\left.9 p_{\phi}\right)^{2}+\left(1 /\left(3 \cdot 44^{2}\right)\right)\left(3 p_{\sigma}-16 p_{\phi}\right)^{2}+(1 /(22 \cdot 44))\left(p_{\sigma}-9 p_{\phi}\right)\left(3 p_{\sigma}-\right.$ $\left.16 p_{\phi}\right)$, we have that

$$
\begin{aligned}
\rho_{a} & \sim e^{-6 \Omega}, \\
\rho_{\phi, \sigma} & \sim e^{-6 \Omega}, \\
\Omega^{\prime 2} & \sim \frac{\Lambda}{3}+\kappa_{\Omega}{ }^{2} e^{-6 \Omega}+b_{\gamma} e^{-3(1+\gamma) \Omega}, \\
b_{\gamma} & =\frac{8}{3} \pi G_{N} \mu_{\gamma} .
\end{aligned}
$$

With the use of these parameters, we find the following ratios:

$$
\begin{aligned}
\frac{\rho_{a}}{\rho_{\phi, \sigma}} & \sim \text { constant, } \\
\frac{\rho_{a}}{\rho_{\gamma}} & \sim e^{3 \Omega(\gamma-1)}, \\
\frac{\rho_{a}}{\Omega^{\prime 2}} & \sim \frac{1}{\kappa_{\Omega}^{2}+(\Lambda / 3) e^{6 \Omega}+b_{\gamma} e^{3(1-\gamma) \Omega}} .
\end{aligned}
$$

We observe that for an expanding Universe the anisotropic density is dominated by the fluid density (with the exception of the stiff fluid) or by the $\Omega^{\prime 2}$ term and then at late times the isotropization is obtained since the above ratios tend to zero.

\section{Case of Interest}

In this section we present the classical solutions for the Hamiltonian density of Lagrangian (13) we have previously built in terms of a new set of variables (16), focusing on three different cases. We start our analysis on the vacuum case, and on the case with a cosmological term $\Lambda$. Finally we will analyze the general case considering matter content and a cosmological term. 
4.1. Vacuum Case. To analyze the vacuum case, we will take in the Hamiltonian density (18) that $\rho_{\gamma}=0$ and $\Lambda=0$, obtaining that

$$
\begin{aligned}
& \mathscr{H}_{I_{\text {vac }}}=\frac{1}{8} e^{-\left(\beta_{1}+\beta_{2}+\beta_{3}\right)}\left[-\Pi_{1}^{2}-\Pi_{2}^{2}-\Pi_{3}^{2}+2 \Pi_{1} \Pi_{2}\right. \\
& \left.\quad+2 \Pi_{1} \Pi_{3}+2 \Pi_{2} \Pi_{3}-\frac{16}{11} \Pi_{\phi}^{2}+\frac{6}{11} \Pi_{\phi} \Pi_{\sigma}-\frac{1}{33} \Pi_{\sigma}^{2}\right] .
\end{aligned}
$$

The Hamilton equations for the coordinates fields $\dot{q}_{i}=$ $\partial \mathscr{H} / \partial P_{i}$ and the corresponding momenta $\dot{P}_{i}=\partial \mathscr{H} / \partial q_{i}$ become

$$
\begin{aligned}
& \dot{\Pi}_{i}=-\mathscr{H} \equiv 0 \Longrightarrow \Pi_{i}=\text { constant, } \\
& \dot{\Pi}_{\phi}=0 \Longrightarrow \Pi_{\phi}=\text { constant, } \\
& \dot{\Pi}_{\sigma}=0 \Longrightarrow \Pi_{\sigma}=\text { constant, } \\
& \beta_{1}^{\prime}=\frac{1}{4} e^{-\left(\beta_{1}+\beta_{2}+\beta_{3}\right)}\left[-\Pi_{1}+\Pi_{2}+\Pi_{3}\right], \\
& \beta_{2}^{\prime}=\frac{1}{4} e^{-\left(\beta_{1}+\beta_{2}+\beta_{3}\right)}\left[-\Pi_{2}+\Pi_{1}+\Pi_{3}\right], \\
& \beta_{3}^{\prime}=\frac{1}{4} e^{-\left(\beta_{1}+\beta_{2}+\beta_{3}\right)}\left[-\Pi_{3}+\Pi_{1}+\Pi_{2}\right], \\
& \phi^{\prime}=\phi_{0} e^{-\left(\beta_{1}+\beta_{2}+\beta_{3}\right)}, \\
& \sigma^{\prime}=\sigma_{0} e^{-\left(\beta_{1}+\beta_{2}+\beta_{3}\right)} .
\end{aligned}
$$

The gravitational momenta are constant by mean of the Hamiltonian constraint, first line in the last equation. In this way, the solution for the sum of $\beta_{i}$ functions becomes

$$
\beta_{1}+\beta_{2}+\beta_{3}=\ln \left[\epsilon \tau+b_{0}\right], \quad \epsilon=b_{1}+b_{2}+b_{3},
$$

with $b_{i}=\Pi_{i}$ and $b_{0}$ being an integration constant. Therefore, Misner variables are expressed as

$$
\begin{aligned}
& \Omega=\ln \left(\epsilon \tau+b_{0}\right)^{1 / 3}, \\
& \beta_{+}=\ln \left(\epsilon \tau+b_{0}\right)^{\left(\epsilon-3 b_{3}\right) / 6 \epsilon}, \\
& \beta_{-}=\ln \left(\epsilon \tau+b_{0}\right)^{\left(b_{3}+2 b_{1}-\epsilon\right) / 2 \sqrt{3} \epsilon},
\end{aligned}
$$

while moduli fields are given by

$$
\begin{gathered}
\phi=\frac{\phi_{0}}{\epsilon} \ln \left(\epsilon \tau+b_{0}\right), \\
\sigma=\frac{\sigma_{0}}{\epsilon} \ln \left(\epsilon \tau+b_{0}\right),
\end{gathered}
$$

where $\phi_{0}=\Pi_{\phi}$ and $\sigma_{0}=\Pi_{\sigma}$. Notice that, in this case, the associated external volume given by $e^{3 \Omega}$ and the moduli fields $\phi$ and $\sigma$ are all logarithmically increasing functions on time, implying for the latter that the internal volume shrinks in size for late times, as expected, while the four-dimensional Universe expands into an isotropic flat spacetime. The parameter $\rho_{a}$ goes to zero at very late times independently of $b_{0}$.
4.2. Cosmological Term $\Lambda$. The corresponding Hamiltonian density becomes

$$
\begin{aligned}
\mathscr{H}_{I} & =\frac{1}{8} e^{-\left(\beta_{1}+\beta_{2}+\beta_{3}\right)}\left[-\Pi_{1}^{2}-\Pi_{2}^{2}-\Pi_{3}^{2}+2 \Pi_{1} \Pi_{2}\right. \\
& +2 \Pi_{1} \Pi_{3}+2 \Pi_{2} \Pi_{3}-\frac{16}{11} \Pi_{\phi}^{2}+\frac{6}{11} \Pi_{\phi} \Pi_{\sigma}-\frac{1}{33} \Pi_{\sigma}^{2} \\
& \left.-16 \Lambda e^{2\left(\beta_{1}+\beta_{2}+\beta_{3}\right)}\right]
\end{aligned}
$$

and corresponding Hamilton's equations are

$$
\begin{aligned}
& \Pi_{1}^{\prime}=\Pi_{2}^{\prime}=\Pi_{3}^{\prime}=4 \Lambda e^{\beta_{1}+\beta_{2}+\beta_{3}}, \\
& \beta_{1}^{\prime}=\frac{1}{4} e^{-\left(\beta_{1}+\beta_{2}+\beta_{3}\right)}\left[-\Pi_{1}+\Pi_{2}+\Pi_{3}\right], \\
& \beta_{2}^{\prime}=\frac{1}{4} e^{-\left(\beta_{1}+\beta_{2}+\beta_{3}\right)}\left[-\Pi_{2}+\Pi_{1}+\Pi_{3}\right], \\
& \beta_{3}^{\prime}=\frac{1}{4} e^{-\left(\beta_{1}+\beta_{2}+\beta_{3}\right)}\left[-\Pi_{3}+\Pi_{1}+\Pi_{2}\right], \\
& \phi^{\prime}=\phi_{0} e^{-\left(\beta_{1}+\beta_{2}+\beta_{3}\right)}, \\
& \sigma^{\prime}=\sigma_{0} e^{-\left(\beta_{1}+\beta_{2}+\beta_{3}\right)},
\end{aligned}
$$

where the constants $\phi_{0}$ and $\sigma_{0}$ are the same as in previous case. To solve the last system of equations we will take the following ansatz:

$$
\Pi_{1}=\Pi_{2}+\delta_{2}=\Pi_{3}+\delta_{3}
$$

with $\delta_{2}$ and $\delta_{3}$ constants. By substituting into Hamiltonian (29) we find a differential equation for the momenta $\Pi_{1}$ given by

$$
\frac{1}{\Lambda} \Pi_{1}^{\prime 2}-3 \Pi_{1}^{2}+\nu \Pi_{1}+\delta_{1}=0
$$

where the corresponding constants are

$$
\begin{gathered}
\nu=2\left(\delta_{2}+\delta_{3}\right), \\
\delta_{1}\left(q_{0}\right)=\left(\delta_{2}-\delta_{3}\right)^{2}-q_{0},
\end{gathered}
$$

with the constant (related to the momenta scalar field)

$$
q_{0}=\frac{16}{11} \Pi_{\phi}^{2}-\frac{6}{11} \Pi_{\phi} \Pi_{\sigma}+\frac{1}{33} \Pi_{\sigma}^{2} .
$$

The solution for $\Pi_{1}$ is thus

$$
\Pi_{1}=\frac{v}{6}+\frac{1}{6} \sqrt{v^{2}+12 \delta_{1}} \cosh (\sqrt{3 \Lambda} \tau) .
$$

Hence, using the last result (35) in the rest of equations in (30) we obtain that

$$
\beta_{1}+\beta_{2}+\beta_{3}=\ln \left(\frac{1}{8} \sqrt{\frac{v^{2}+12 \delta_{1}}{3 \Lambda}} \sinh (\sqrt{3 \Lambda} \tau)\right),
$$


and the corresponding solutions to the moduli fields are

$$
\begin{aligned}
& \phi=\frac{8 \phi_{2}}{\sqrt{\nu^{2}+12 \delta_{1}}} \ln \left(\tanh \left(\frac{\sqrt{3 \Lambda}}{2} \tau\right)\right), \\
& \sigma=\frac{8 \sigma_{2}}{\sqrt{\nu^{2}+12 \delta_{1}}} \ln \left(\tanh \left(\frac{\sqrt{3 \Lambda}}{2} \tau\right)\right) .
\end{aligned}
$$

With the last results we see that the solutions to the Misner variables $\left(\Omega, \beta_{+}\right)$are given by

$$
\begin{aligned}
& \Omega=\frac{1}{3} \ln \left(\frac{1}{8} \sqrt{\frac{\nu^{2}+12 \delta_{1}}{3 \Lambda}} \sinh (\sqrt{3 \Lambda} \tau)\right), \\
& \beta_{+}=\frac{2}{3} \frac{\left(\delta_{2}-2 \delta_{3}\right)}{\sqrt{\nu^{2}+12 \delta_{1}}} \ln \tanh \left(\frac{\sqrt{3 \Lambda}}{2} \tau\right), \\
& \beta_{-}=-\frac{2}{\sqrt{3}} \frac{\delta_{2}}{\sqrt{v^{2}+12 \delta_{1}}} \ln \tanh \left(\frac{\sqrt{3 \Lambda}}{2} \tau\right) .
\end{aligned}
$$

and the isotropization parameter $\rho_{a}$ is given by

$$
\rho_{a}=\frac{\Lambda}{3} \frac{\left(\delta_{2}-2 \delta_{3}\right)^{2}+\delta_{2}^{2}}{v^{2}+12\left(\left(\delta_{2}-\delta_{3}\right)^{2}-q_{0}\right)} \operatorname{coth}^{2}\left(\frac{\sqrt{3 \Lambda}}{2} \tau\right) .
$$

Notice that the external four-dimensional volume expands as time $\tau$ runs and it is affected whether $q_{0}$ is positive or negative. This in turn determines how fast the moduli fields evolve in relation to each other (i.e., whether $\Pi_{\phi}$ is larger or smaller than $\Pi_{\sigma}$ ). Therefore, isotropization is reached independently of the values of $q_{0}$ but expansion of the Universe (and shrinking of the internal volume) is affected by it.

4.3. Matter Content and Cosmological Term. For this case, the corresponding Hamiltonian density becomes (18). So, using the Hamilton equation, we can see that the momenta associated with the scalars fields $\phi$ and $\sigma$ are constants, and we will label these constants by $\phi_{1}$ and $\sigma_{1}$, respectively:

$$
\begin{aligned}
\Pi_{1}^{\prime} & =\Pi_{2}^{\prime}=\Pi_{3}^{\prime} \\
& =4 \Lambda e^{\beta_{1}+\beta_{2}+\beta_{3}}+16 \pi G(1-\gamma) \rho_{\gamma} e^{-\gamma\left(\beta_{1}+\beta_{2}+\beta_{3}\right)}, \\
\beta_{1}^{\prime} & =\frac{1}{4} e^{-\left(\beta_{1}+\beta_{2}+\beta_{3}\right)}\left[-\Pi_{1}+\Pi_{2}+\Pi_{3}\right], \\
\beta_{2}^{\prime} & =\frac{1}{4} e^{-\left(\beta_{1}+\beta_{2}+\beta_{3}\right)}\left[-\Pi_{2}+\Pi_{1}+\Pi_{3}\right] \\
\beta_{3}^{\prime} & =\frac{1}{4} e^{-\left(\beta_{1}+\beta_{2}+\beta_{3}\right)}\left[-\Pi_{3}+\Pi_{1}+\Pi_{2}\right] \\
\phi^{\prime} & =\phi_{2} e^{-\left(\beta_{1}+\beta_{2}+\beta_{3}\right)} \\
\sigma^{\prime} & =\sigma_{2} e^{-\left(\beta_{1}+\beta_{2}+\beta_{3}\right)}
\end{aligned}
$$

Follow the same steps as in Section 4.2, we see that it is possible to relate the momenta associated with $\beta_{1}, \beta_{2}$, and $\beta_{3}$ in the following way:

$$
\Pi_{1}=\Pi_{2}+k_{1}=\Pi_{3}+k_{2}
$$

Also, the differential equation for fields $\phi$ and $\sigma$ can be reduced to quadrature as

$$
\begin{aligned}
& \phi=\phi_{2} \int e^{-\left(\beta_{1}+\beta_{2}+\beta_{3}\right)} d \tau, \\
& \sigma=\sigma_{2} \int e^{-\left(\beta_{1}+\beta_{2}+\beta_{3}\right)} d \tau .
\end{aligned}
$$

We proceed to consider specific values for $\gamma$.

4.3.1. $\gamma= \pm 1$ Cases. Let us introduce the generic parameter

$$
\lambda= \begin{cases}4 \Lambda, & \gamma=1 \\ 4 \Lambda+32 \pi G \rho_{-1}, & \gamma=-1\end{cases}
$$

for which $\Pi_{1}^{\prime}=\lambda e^{\beta_{1}+\beta_{2}+\beta_{3}}$. Substituting this into Hamiltonian (18) we find the differential equation for the momenta $\Pi_{1}$ as

$$
\frac{4}{\lambda} \Pi_{1}^{\prime 2}-3 \Pi_{1}^{2}+v \Pi_{1}+\delta_{1}=0
$$

with the solution given by $\Pi_{1}$ is

$$
\Pi_{1}=\frac{v}{6}+\frac{1}{6} \sqrt{\nu^{2}+12 \delta_{1}} \cosh \left(\frac{\sqrt{3 \lambda}}{2} \tau\right) .
$$

On the other hand, using the last result (45) we obtain from Hamilton equations that

$$
\beta_{1}+\beta_{2}+\beta_{3}=\ln \left(\frac{1}{4} \sqrt{\frac{\delta_{3}+12 \delta_{1}}{3 \lambda}} \sinh \left(\frac{\sqrt{3 \lambda}}{2} \tau\right)\right) .
$$

The corresponding solutions to the moduli fields (42) can be found as

$$
\begin{gathered}
\phi=\frac{8 \phi_{2}}{\sqrt{\nu^{2}+12 \delta_{1}}} \ln \tanh \left(\frac{\sqrt{3 \lambda}}{4} \tau\right), \\
\sigma=\frac{8 \sigma_{2}}{\sqrt{\nu^{2}+12 \delta_{1}}} \ln \tanh \left(\frac{\sqrt{3 \lambda}}{4} \tau\right) .
\end{gathered}
$$


The solutions to Misner variables $(16),\left(\Omega, \beta_{ \pm}\right)$, are given by

$$
\begin{aligned}
\Omega= & \frac{1}{3} \ln \left(\frac{1}{4} \sqrt{\frac{\delta^{2}+12 \delta_{1}}{3 \lambda}} \sinh \left(\frac{\sqrt{3 \lambda}}{2} \tau\right)\right), \\
\beta_{+}= & \frac{2}{3} \frac{\left(\delta_{2}-2 \delta_{3}\right)}{\sqrt{\nu^{2}+12 \delta_{1}}} \ln \tanh \left(\frac{\sqrt{3 \lambda}}{2} \tau\right) \\
& +\frac{1}{6} \ln \left(\frac{1}{4} \sqrt{\frac{\nu^{2}+12 \delta_{1}}{3 \lambda}}\right), \\
\beta_{-}= & -\frac{2}{\sqrt{3}} \frac{\delta_{2}}{\sqrt{\nu^{2}+12 \delta_{1}}} \ln \tanh \left(\frac{\sqrt{3 \lambda}}{2} \tau\right) \\
& -\frac{1}{2 \sqrt{3}} \ln \left(\frac{1}{4} \sqrt{\frac{\nu^{2}+12 \delta_{1}}{3 \lambda}}\right) .
\end{aligned}
$$

It is remarkable that (42) for the fields $\phi$ and $\sigma$ are maintained for all Bianchi Class A models, and in particular, when we use the gauge $N=e^{\beta_{1}+\beta_{2}+\beta_{3}}$, the solutions for these fields are independent of the cosmological models, whose solutions are $\phi=\left(\phi_{0} / 8\right) t$ and $\sigma=\left(\sigma_{0} / 8\right) t$.

After studying the $\gamma=0$ case we will comment on the physical significance of these solutions.

4.3.2. $\gamma=0$ Case. The Hamilton equations are

$$
\begin{aligned}
& \Pi_{1}^{\prime}=\Pi_{2}^{\prime}=\Pi_{3}^{\prime}=4 \Lambda e^{\beta_{1}+\beta_{2}+\beta_{3}}+\alpha_{0}, \quad \alpha_{0}=16 \pi G \rho_{0} \\
& \Pi_{\phi}^{\prime}=0, \quad \Pi_{\phi}=\phi_{1}=c t e
\end{aligned}
$$

from which the momenta $\Pi_{1}$ fulfil the equation

$$
\frac{1}{\Lambda} \Pi_{1}^{\prime 2}-3 \Pi_{1}^{2}+\mu \Pi_{1}+\xi_{1}=0
$$

where

$$
\xi_{1}=\delta_{1}-\frac{\alpha_{0}^{2}}{\Lambda}
$$

The solution is given by

$$
\Pi_{1}=\frac{v}{6}+\frac{1}{6} \sqrt{v^{2}+12 \xi_{1}} \cosh (\sqrt{3 \Lambda} \tau)
$$

Using this result in the corresponding Hamilton equations, we obtain that

$$
\beta_{1}+\beta_{2}+\beta_{3}=\ln \left(\frac{1}{8} \sqrt{\frac{\nu^{2}+12 \xi_{1}}{3 \Lambda}} \sinh (\sqrt{3 \Lambda} \tau)\right),
$$

and the corresponding solutions to the dilaton field $\phi$ and the moduli field $\sigma$ are given by

$$
\begin{gathered}
\phi=\frac{8 \phi_{2}}{\sqrt{\nu^{2}+12 \xi_{1}}} \ln \tanh \left(\frac{\sqrt{3 \Lambda}}{2} \tau\right), \\
\sigma=\frac{8 \sigma_{2}}{\sqrt{\nu^{2}+12 \xi_{1}}} \ln \tanh \left(\frac{\sqrt{3 \Lambda}}{2} \tau\right) .
\end{gathered}
$$

So, the solutions to the Misner variables $\left(\Omega, \beta_{ \pm}\right)$are

$$
\begin{aligned}
& \Omega=\frac{1}{3} \ln \left(\frac{1}{8} \sqrt{\frac{\nu^{2}+12 \xi_{1}}{3 \Lambda}} \sinh (\sqrt{3 \Lambda} \tau)\right), \\
& \beta_{+}=\frac{2}{3} \frac{\delta_{2}-2 \delta_{3}}{\sqrt{\nu^{2}+12 \xi_{1}}} \ln \tanh \left(\frac{\sqrt{3 \Lambda}}{2} \tau\right), \\
& \beta_{-}=-\frac{2}{\sqrt{3}} \frac{\delta_{2}}{\sqrt{\nu^{2}+12 \xi_{1}}} \ln \tanh \left(\frac{\sqrt{3 \Lambda}}{2} \tau\right) .
\end{aligned}
$$

For both solutions, $\gamma=0, \pm 1$, we also observe that, for positive (negative) $q_{0}$, the Universe expands slower (faster) than in the absence of extra dimensions while the internal space shrinks faster (slower) for positive (negative) $q_{0}$. In this case, isotropization parameters are also accordingly affected by $q_{0}$, through relations (34).

So far, our analysis has been completely classic. Now, in the next section we deal with the quantum scheme and we are going to solve the WDW equation in standard quantum cosmology.

\section{Quantum Scheme}

Solutions to the Wheeler-DeWitt (WDW) equation dealing with different problems have been extensively used in the literature. For example, the important quest of finding a typical wave function for the Universe was nicely addressed in [39], while in [40] there appears an excellent summary concerning the problem of how a Universe emerged from a big bang singularity, whihc cannot longer be neglected in the GUT epoch. On the other hand, the best candidates for quantum solutions become those that have a damping behavior with respect to the scale factor, represented in our model with the $\Omega$ parameter, in the sense that we obtain a good classical solution using the WKB approximation in any scenario in the evolution of our Universe [41]. The WDW equation for this model is achieved by replacing the momenta $\Pi_{q^{\mu}}=-i \partial_{q^{\mu}}$, associated with the Misner variables $\left(\Omega, \beta_{+}, \beta_{-}\right)$ and the moduli fields $(\phi, \sigma)$ in Hamiltonian (15). The factor $e^{-3 \Omega}$ may be factor ordered with $\widehat{\Pi}_{\Omega}$ in many ways. Hartle and Hawking [41] have suggested what might be called a semigeneral factor ordering which in this case would order $e^{-3 \Omega} \widehat{\Pi}_{\Omega}^{2}$ as

$$
-e^{-(3-Q) \Omega} \partial_{\Omega} e^{-Q \Omega} \partial_{\Omega}=-e^{-3 \Omega} \partial_{\Omega}^{2}+Q e^{-3 \Omega} \partial_{\Omega},
$$

where $Q$ is any real constant that measure the ambiguity in the factor ordering in the variable $\Omega$ and the corresponding momenta. We will assume in the following this factor ordering for the Wheeler-DeWitt equation, which becomes

$$
\begin{gathered}
\square \Psi+Q \frac{\partial \Psi}{\partial \Omega}-\frac{48}{11} \frac{\partial^{2} \Psi}{\partial \phi^{2}}-\frac{1}{11} \frac{\partial^{2} \Psi}{\partial \sigma^{2}}+\frac{18}{11} \frac{\partial^{2} \Psi}{\partial \phi \partial \sigma} \\
-\left[b_{\gamma} e^{3(1-\gamma) \Omega}+48 \Lambda e^{6 \Omega}\right] \Psi=0,
\end{gathered}
$$


where $\square$ is the three-dimensional d'Lambertian in the $\ell^{\mu}=$ $\left(\Omega, \beta_{+}, \beta_{-}\right)$coordinates, with signature $(-++)$. On the other hand, we could interpret the WDW equation (57) as a timereparametrization invariance of the wave function $\Psi$. At a glance, we can see that the WDW equation is static; this can be understood as the problem of time in standard quantum cosmology. We can avoid this problem by measuring the physical time with respect a kind of time variable anchored within the system, which means that we could understand the WDW equation as a correlation between the physical time and a fictitious time $[42,43]$. When we introduce the ansatz

$$
\Psi=\Phi(\phi, \sigma) \psi\left(\Omega, \beta_{ \pm}\right),
$$

in (57), we obtain the general set of differential equations (under the assumed factor ordering):

$$
\begin{array}{r}
\square \psi+Q \frac{\partial \psi}{\partial \Omega}-\left[b_{\gamma} e^{3(1-\gamma) \Omega}+48 \Lambda e^{6 \Omega}-\frac{\mu^{2}}{5}\right] \psi=0, \\
\frac{48}{11} \frac{\partial^{2} \Phi}{\partial \phi^{2}}+\frac{1}{11} \frac{\partial^{2} \Phi}{\partial \sigma^{2}}-\frac{18}{11} \frac{\partial^{2} \Phi}{\partial \phi \partial \sigma}+\mu^{2} \Phi=0,
\end{array}
$$

where we choose the separation constant $\mu^{2} / 5$ for convenience to reduce the second equation. The solution to the hyperbolic partial differential equation (60) is given by

$$
\begin{aligned}
\Phi(\phi, \sigma)= & C_{1} \sin \left(C_{3} \phi+C_{4} \sigma+C_{5}\right) \\
& +C_{2} \cos \left(C_{3} \phi+C_{4} \sigma+C_{5}\right),
\end{aligned}
$$

where $\left\{C_{i}\right\}_{i=1}^{5}$ are integration constants and they are in terms of $\mu$. We claim that this solution is the same for all Bianchi Class A cosmological models, because the Hamiltonian operator in (57) can be written in separated way as $\widehat{H}\left(\Omega, \beta_{ \pm}, \phi, \sigma\right) \Psi=\widehat{H}_{g}\left(\Omega, \beta_{ \pm}\right) \Psi+\widehat{H}_{m}(\phi, \sigma) \Psi=0$, where $\widehat{H}_{g}$ y $\widehat{H}_{m}$ represents the Hamiltonian to gravitational sector and the moduli fields, respectively. To solve (59), we now set $\psi\left(\Omega, \beta_{ \pm}\right)=\mathscr{A}(\Omega) \mathscr{B}_{1}\left(\beta_{+}\right) \mathscr{B}_{2}\left(\beta_{-}\right)$, obtaining the following set of ordinary differential equations:

$$
\begin{aligned}
& \frac{d^{2} \mathscr{A}}{d \Omega^{2}}-Q \frac{d \mathscr{A}}{d \Omega}+\left[b_{\gamma} e^{-3(\gamma-1) \Omega}+48 \Lambda e^{6 \Omega}+\sigma^{2}\right] \mathscr{A}=0, \\
& \frac{d^{2} \mathscr{B}_{1}}{d \beta_{+}^{2}}+a_{2}^{2} \mathscr{B}_{1}=0 \Longrightarrow \\
& \mathscr{B}_{1}=\eta_{1} e^{i a_{2} \beta_{+}}+\eta_{2} e^{-i a_{2} \beta_{+}}, \\
& \frac{d^{2} \mathscr{B}_{2}}{d \beta_{-}^{2}}+a_{3}^{2} \mathscr{B}_{2}=0 \Longrightarrow \\
& \mathscr{B}_{2}=c_{1} e^{i a_{3} \beta_{-}}+c_{2} e^{-i a_{3} \beta_{-}},
\end{aligned}
$$

where $\sigma^{2}=a_{1}^{2}+a_{2}^{2}+a_{3}^{2}$ and $c_{i}$ and $\eta_{i}$ are constants. We now focus on the $\Omega$ dependent part of the WDW equation. We solve this equation when $\Lambda=0$ and $\Lambda \neq 0$.

(1) For the case with null cosmological constant and $\gamma \neq 1$,

$$
\frac{d^{2} \mathscr{A}}{d \Omega^{2}}-Q \frac{d \mathscr{A}}{d \Omega}+\left[b_{\gamma} e^{-3(1-\gamma) \Omega}+\sigma^{2}\right] \mathscr{A}=0
$$

and by using the change of variable,

$$
z=\frac{\sqrt{b_{\gamma}}}{p} e^{-(3 / 2)(\gamma-1) \Omega},
$$

where $p$ is a parameter to be determined, we have

$$
\begin{aligned}
\frac{d \mathscr{A}}{d \Omega} & =\frac{d \mathscr{A}}{d z} \frac{d z}{d \Omega}=-\frac{3}{2}(\gamma-1) z \frac{d \mathscr{A}}{d z}, \\
\frac{d^{2} \mathscr{A}}{d \Omega^{2}} & =\frac{9}{4}(\gamma-1)^{2} z^{2} \frac{d^{2} \mathscr{A}}{d z^{2}}+\frac{9}{4}(\gamma-1)^{2} z \frac{d \mathscr{A}}{d z} .
\end{aligned}
$$

Hence, we arrive at the equation

$$
\begin{aligned}
\frac{9}{4}(\gamma-1)^{2} z^{2} \frac{d^{2} \mathscr{A}}{d z^{2}}+\frac{9}{4}(\gamma-1)^{2} z \frac{d \mathscr{A}}{d z} \\
-\frac{3}{2} Q(\gamma-1) z \frac{d \mathscr{A}}{d z}+\left[p^{2} z^{2}+\sigma^{2}\right]=0,
\end{aligned}
$$

where we have assumed that $\mathscr{A}$ is of the form [44]

$$
\mathscr{A}=z^{q \mathrm{Q}} \Phi(z),
$$

with $q$ being yet to be determined. We thus get, after substituting in (66),

$$
\begin{aligned}
& \frac{9}{4}(\gamma-1)^{2} z^{q Q}\left[z^{2} \frac{d^{2} \Phi}{d z^{2}}+z(1+2 q Q\right. \\
& \left.+\frac{2}{3} \frac{Q}{\gamma-1}\right) \frac{d \Phi}{d z}+\left(\frac{4 p^{2}}{9(\gamma-1)^{2}} z^{2}\right. \\
& \left.\left.+Q^{2}\left\{q^{2}+\frac{2}{3} \frac{q}{\gamma-1}\right\}+\frac{4 \sigma^{2}}{9(\gamma-1)^{2}}\right) \Phi\right]=0,
\end{aligned}
$$

which can be written as

$$
\begin{aligned}
& z^{2} \frac{d^{2} \Phi}{d z^{2}}+z \frac{d \Phi}{d z}+\left[z^{2}-\frac{1}{9(\gamma-1)^{2}}\left(Q^{2}-4 \sigma^{2}\right)\right] \Phi \\
& \quad=0
\end{aligned}
$$

which is the Bessel differential equation for the function $\Phi$ when $p$ and $q$ are fixed to

$$
\begin{aligned}
& q=-\frac{1}{3(\gamma-1)}, \\
& p=\frac{3}{2}|\gamma-1|,
\end{aligned}
$$

which in turn means that transformations (64) and (67) are

$$
\begin{aligned}
z & =\frac{2 \sqrt{b_{\gamma}}}{3|\gamma-1|} e^{-(3 / 2)(\gamma-1) \Omega}, \\
\mathscr{A} & =z^{-(\mathrm{Q} / 3(\gamma-1)) \Phi(z)} .
\end{aligned}
$$


Hence, the solution for (63) is of the form

$$
\begin{gathered}
\mathscr{A}_{\gamma}=c_{\gamma}\left(\frac{2 \sqrt{b_{\gamma}}}{3|\gamma-1|} e^{-(3 / 2)(\gamma-1) \Omega}\right)^{-\mathrm{Q} / 3(\gamma-1)} \\
\cdot Z_{i \nu}\left(\frac{2 \sqrt{b_{\gamma}}}{3|\gamma-1|} e^{-(3 / 2)(\gamma-1) \Omega}\right)
\end{gathered}
$$

with

$$
\nu= \pm \sqrt{\frac{1}{9(\gamma-1)^{2}}\left(4 \sigma^{2}-Q^{2}\right)},
$$

where $Z_{i v}=J_{i v}$ is the ordinary Bessel function with imaginary order. For the particular case when the factor ordering is zero, we can easily construct a wave packet $[41,45,46]$ using the identity:

$$
\int_{-\infty}^{\infty} \operatorname{sech}\left(\frac{\pi \eta}{2}\right) J_{i \eta}(z) d \eta=2 \sin (z),
$$

so, the total wave function becomes

$$
\begin{aligned}
\Psi\left(\Omega, \beta_{ \pm}, \phi, \sigma\right)= & \Phi(\phi, \sigma) \\
& \cdot \sin \left(\frac{2 \sqrt{b_{\gamma}}}{3|\gamma-1|} e^{-(3 / 2)(\gamma-1) \Omega}\right) \\
& \cdot\left[\eta_{1} e^{i a_{2} \beta_{+}}+\eta_{2} e^{-i a_{2} \beta_{+}}\right] \\
& \cdot\left[c_{1} e^{i a_{3} \beta_{-}}+c_{2} e^{-i a_{3} \beta_{-}}\right] .
\end{aligned}
$$

Notice that the influence of extra dimensions through the presence of the moduli $\phi$ and $\sigma$ appears in the solution in the amplitude of the wave function. We will comment on this later on.

(2) Case with null cosmological constant and $\gamma=1$. For this case we have

$$
\frac{d^{2} \mathscr{A}_{1}}{d \Omega^{2}}-Q \frac{d \mathscr{A}_{1}}{d \Omega}+\sigma_{1}^{2} \mathscr{A}_{1}=0, \quad \sigma_{1}^{2}=b_{1}+\sigma^{2}
$$

whose solution is

$$
\mathscr{A}_{1}=A_{1} e^{\left(\left(\mathrm{Q}+\sqrt{\mathrm{Q}^{2}+4 \sigma_{1}^{2}}\right) / 2\right) \Omega}+A_{2} e^{\left(\left(\mathrm{Q}-\sqrt{\mathrm{Q}^{2}+4 \sigma_{1}^{2}}\right) / 2\right) \Omega} .
$$

So, the total wave function becomes

$$
\begin{gathered}
\Psi\left(\Omega, \beta_{ \pm}, \phi, \sigma\right)=\Phi(\phi, \sigma) \\
\cdot\left[A_{1} e^{\left(\left(\mathrm{Q}+\sqrt{\mathrm{Q}^{2}+4 \sigma_{1}^{2}}\right) / 2\right) \Omega}+A_{2} e^{\left(\left(\mathrm{Q}-\sqrt{Q^{2}+4 \sigma_{1}^{2}}\right) / 2\right) \Omega}\right] \\
\cdot\left[\eta_{1} e^{i a_{2} \beta_{+}}+\eta_{2} e^{-i a_{2} \beta_{+}}\right]\left[c_{1} e^{i a_{3} \beta_{-}}+c_{2} e^{-i a_{3} \beta_{-}}\right] .
\end{gathered}
$$

(3) If we include the cosmological constant term for this particular case, we have [44]

$$
\mathscr{A}=e^{(\mathrm{Q} / 2) \Omega} Z_{\nu}\left(4 \sqrt{\frac{\Lambda}{3}} e^{3 \Omega}\right), \quad v= \pm \frac{1}{6} \sqrt{Q^{2}+4 \sigma_{1}^{2}},
$$

where $\Lambda>0$ in order to have ordinary Bessel functions as solutions; in other case, we will have the modified Bessel function. When the factor ordering parameter $Q$ equals zero, we have the same generic Bessel functions as solutions, having the imaginary order $\nu= \pm i \sqrt{\sigma_{1}^{2}} / 3$.

(4) $\gamma=-1$ and $\Lambda \neq 0$ and any factor ordering $Q$ :

$$
\begin{aligned}
\frac{d^{2} \mathscr{A}_{-1}}{d \Omega^{2}}-Q \frac{d \mathscr{A}_{-1}}{d \Omega}+\left[d_{-1} e^{6 \Omega}-\sigma^{2}\right] \mathscr{A}_{-1} & =0, \\
d_{-1} & =48 \mu_{-1}+48 \Lambda
\end{aligned}
$$

with the solution

$$
\begin{array}{r}
\mathscr{A}_{-1}=\left(\frac{\sqrt{d_{-1}}}{3} e^{3 \Omega}\right)^{\mathrm{Q} / 6} Z_{\nu}\left(\frac{\sqrt{d_{-1}}}{3} e^{3 \Omega}\right), \\
\nu= \pm \frac{1}{6} \sqrt{\mathrm{Q}^{2}+4 \sigma^{2}},
\end{array}
$$

where $Z_{\nu}$ is a generic Bessel function. When $b_{-1}>0$, we have ordinary Bessel functions; in other case, we have modified Bessel functions.

(5) $\gamma=0, \Lambda \neq 0$, and factor ordering $Q=0$

$$
\frac{d^{2} \mathscr{A}_{0}}{d \Omega^{2}}+\left(48 \Lambda e^{6 \Omega}+b_{0} e^{3 \Omega}+\sigma^{2}\right) \mathscr{A}_{0}=0, \quad b_{0}=48 \mu_{0}
$$

with the solution

$$
\begin{aligned}
\mathscr{A}_{0} & =e^{-3 \Omega / 2}\left[D_{1} M_{-i b_{0} / 24 \sqrt{3 \Lambda},-i \sigma / 3}\left(\frac{8 i e^{3 \Omega} \sqrt{\Lambda}}{\sqrt{3}}\right)\right. \\
& \left.+D_{2} W_{-i b_{0} / 24 \sqrt{3 \Lambda},-i \sigma / 3}\left(\frac{8 i e^{3 \Omega} \sqrt{\Lambda}}{\sqrt{3}}\right)\right],
\end{aligned}
$$

where $M_{k, p}$ And $W_{k, p}$ are Whittaker functions and $D_{i}$ are integration constants.

\section{Final Remarks}

In this work we have explored a compactification of a tendimensional gravity theory coupled with a time-dependent dilaton into a time-dependent six-dimensional torus. The effective theory which emerges through this process resembles the Einstein frame to that described by the Bianchi I model. By incorporating the barotropic matter and cosmological content and by using the analytical procedure of Hamilton equation of classical mechanics, in appropriate coordinates, we found the classical solution for the anisotropic Bianchi type I cosmological models. In particular, the Bianchi type I is completely solved without using a particular gauge. With these solutions we can validate our qualitative analysis on isotropization of the cosmological model, implying that the volume becomes larger in the corresponding time evolution.

We find that, for all cases, the Universe expansion is gathered as time runs while the internal space shrinks. Qualitatively this model shows us that extra dimensions are 
forced to decrease its volume for an expanding Universe. Also we notice that the presence of extra dimensions affects how fast the Universe (with matter) expands through the presence of a constant related to the moduli momenta. This is not unexpected since we are not considering a potential for the moduli, which implies that they are not stabilized and consequently an effective model should only take into account their constant momenta. We observe that isotropization is not affected in the cases without matter, but in the matter presence, isotropization can be favored or retarded according to how fast the moduli evolve with respect to each other.

It could be interesting to study other types of matter in this context, beyond the barotropic matter. For instance, the Chaplygin gas, with a proper time, characteristic to this matter, leads to the presence of singularities types I, II, III, and IV (generalizations to these models are presented in [47-49]) which also appear in the phantom scenario to dark/energy matter. In our case, since the matter we are considering is barotropic, initial singularities of these types do not emerge from our analysis, implying also that phantom fields are absent. It is important to remark that this model is a very simplified one in the sense that we do not consider the presence of moduli-dependent matter and we do not analyze under which conditions inflation is present or how it starts. We plan to study this important task in a different work.

Concerning the quantum scheme we can observe that this anisotropic model is completely integrable without employing numerical methods, similar solutions to partial differential equation into the gravitational variables have been found in [50], and we obtain that the solutions in the moduli fields are the same for all Bianchi Class A cosmological models, because the Hamiltonian operator in (57) can be written in separated way as $\widehat{H}\left(\Omega, \beta_{ \pm}, \phi, \sigma\right) \Psi=\widehat{H}_{g}\left(\Omega, \beta_{ \pm}\right) \Psi+$ $\widehat{H}_{m}(\phi, \sigma) \Psi=0$, where $\widehat{H}_{g}$ y $\widehat{H}_{m}$ represents the Hamiltonian to gravitational sector and the moduli fields, respectively, with the full wave function given by $\Psi=\Phi(\phi, \sigma) \Theta\left(\Omega, \beta_{ \pm}\right)$. In order to have the best candidates for quantum solutions become those that have a damping behavior with respect to the scale factor, represented in our model with $\Omega$ parameter, in this way we will drop in the full solution the modified Bessel function $I_{\nu}(z)$ or Bessel function $Y_{\nu}(z)$, with $v$ being the order of these functions.

We can observe that the quantum solution in the $\Omega$ sector is similar to the corresponding FRW cosmological model, found in different schemes [12, 22, 23, 32, 43]. Also, similar analysis based on a Bianchi type II model can be found in recent published paper by two authors of this work [51].

The presence of extra dimensions could have a stronger influence on the isotropization process by having a modulidependent potential, which can be gathered by turning on extra fields in the internal space, called fluxes. It will be interesting to consider a more complete compactification process in which all moduli are considered as time-dependent fields as well as time-dependent fluxes. We leave these important analyses for future work.

\section{Appendix}

\section{Dimensional Reduction}

With the purpose to be consistent, here we present the main ideas to dimensionally reduce action (1). By the use of the conformal transformation

$$
\widehat{G}_{M N}=e^{\Phi / 2} G_{M N}^{E},
$$

action (1) can be written as

$$
\begin{aligned}
S= & \frac{1}{2 \kappa_{10}^{2}} \int d^{10} X \sqrt{-\widehat{G}}\left(e^{\Phi / 2} \widehat{\mathscr{R}}+4 G^{E_{M N}} \nabla_{M} \Phi \nabla_{N} \Phi\right) \\
& +\int d^{10} X \sqrt{-G^{E}} e^{5 \Phi / 2} \mathscr{L}_{\text {matt }},
\end{aligned}
$$

where the ten-dimensional scalar curvature $\widehat{\mathscr{R}}$ transforms according to the conformal transformation as

$$
\begin{aligned}
\widehat{\mathscr{R}} & =e^{-\Phi / 2}\left(\mathscr{R}^{E}-\frac{9}{2} G^{E_{M N}} \nabla_{M} \nabla_{N} \Phi\right. \\
& \left.-\frac{9}{2} G^{E_{M N}} \nabla_{M} \Phi \nabla_{N} \Phi\right) .
\end{aligned}
$$

By substituting expression (A.3) in (A.2) we obtain

$$
\begin{aligned}
S= & \frac{1}{2 \kappa_{10}^{2}} \int d^{10} X \sqrt{-G^{E}}\left(\mathscr{R}^{E}-\frac{9}{2} G^{E_{M N}} \nabla_{M} \nabla_{N} \Phi\right. \\
& \left.-\frac{1}{2} G^{E_{M N}} \nabla_{M} \Phi \nabla_{N} \Phi\right)+\int d^{10} X \sqrt{-G^{E}} e^{5 \Phi / 2} \mathscr{L}_{\text {matt }}
\end{aligned}
$$

The last expression is the ten-dimensional action in the Einstein frame. Expressing the metric determinant in fourdimensions in terms of the moduli field $\sigma$ we have

$$
\operatorname{det} \widehat{G}_{M N}=\widehat{G}=e^{-12 \sigma} \widehat{g} \text {. }
$$

By substituting the last expression (A.5) in (1) and considering that

$$
\widehat{\mathscr{R}}^{(10)}=\widehat{\mathscr{R}}^{(4)}-42 \widehat{g}^{\mu \nu} \nabla_{\mu} \sigma \nabla_{\nu} \sigma+12 \widehat{g}^{\mu \nu} \nabla_{\mu} \nabla_{\nu} \sigma,
$$

we obtain that

$$
\begin{aligned}
S= & \frac{1}{2 \kappa_{10}^{2}} \int d^{4} x d^{6} y \sqrt{-\widehat{g}} e^{-6 \sigma} e^{-2 \Phi}\left[\widehat{\mathscr{R}}^{(4)}\right. \\
& \left.-42 \widehat{g}^{\mu \nu} \nabla_{\mu} \sigma \nabla_{\nu} \sigma+12 \widehat{g}^{\mu \nu} \nabla_{\mu} \nabla_{\nu} \sigma+4 \widehat{g}^{\mu \nu} \nabla_{\mu} \Phi \nabla_{\nu} \Phi\right] .
\end{aligned}
$$

Let us redefine in the last expression the dilaton field $\Phi$ as

$$
\Phi=\phi-\frac{1}{2} \ln (\widehat{V})
$$

where $\widehat{V}=\int d^{6} y$. So, expression (A.7) can be written as

$$
\begin{aligned}
S= & \frac{1}{2 \kappa_{10}^{2}} \int d^{4} x \sqrt{-\widehat{g}} e^{-2(\phi+3 \sigma)}\left[\widehat{\mathscr{R}}^{(4)}-42 \widehat{\mathfrak{g}}^{\mu \nu} \nabla_{\mu} \sigma \nabla_{\nu} \sigma\right. \\
& \left.+12 \widehat{g}^{\mu \nu} \nabla_{\mu} \nabla_{\nu} \sigma+4 \widehat{g}^{\mu \nu} \nabla_{\mu} \phi \nabla_{\nu} \phi\right] .
\end{aligned}
$$


The four-dimensional metric $\widehat{g}_{\mu \nu}$ means that the metric is in the string frame, and we should take a conformal transformation linking the String and Einstein frames. We label by $g_{\mu \nu}$ the metric of the external space in the Einstein frame; this conformal transformation is given by

$$
\widehat{\mathfrak{g}}_{\mu \nu}=e^{2 \Theta} g_{\mu \nu}
$$

which after some algebra gives the four-dimensional scalar curvature:

$$
\widehat{\mathscr{R}}^{(4)}=e^{-2 \Theta}\left(\mathscr{R}^{(4)}-6 g^{\mu \nu} \nabla_{\mu} \nabla_{\nu} \Theta-6 g^{\mu \nu} \nabla_{\mu} \Theta \nabla_{\nu} \Theta\right),
$$

where the function $\Theta$ is given by the transformation:

$$
\Theta=\phi+3 \sigma+\ln \left(\frac{\kappa_{10}^{2}}{\kappa_{4}^{2}}\right) .
$$

Now, we must replace expressions (A.10), (A.11), and (A.12) in expression (A.9) and we find

$$
\begin{aligned}
S= & \frac{1}{2 \kappa_{4}^{2}} \int d^{4} x \sqrt{-g}\left(\mathscr{R}-6 g^{\mu \nu} \nabla_{\mu} \nabla_{\nu} \phi-6 g^{\mu \nu} \nabla_{\mu} \nabla_{\nu} \sigma\right. \\
& \left.-2 g^{\mu \nu} \nabla_{\mu} \phi \nabla_{\nu} \phi-96 g^{\mu \nu} \nabla_{\mu} \sigma \nabla_{\nu} \sigma-36 g^{\mu \nu} \nabla_{\mu} \phi \nabla_{\nu} \sigma\right) .
\end{aligned}
$$

At first glance, it is important to clarify one point related to the stress-energy tensor which has the matrix form (6); this tensor belongs to the String frame. In order to write the stressenergy tensor in the Einstein frame we need to work with the last integrand of expression (1). So, after taking the variation with respect to the ten-dimensional metric and opening the expression we see that

$$
\begin{aligned}
\int d^{10} X \sqrt{-\widehat{G}} e^{-2 \Phi} \kappa_{10}^{2} e^{2 \Phi} \widehat{T}_{M N} \widehat{G}^{M N} \\
=\int d^{4} x \sqrt{-g} e^{2(\Theta+\phi)} \widehat{T}_{M N} \widehat{G}^{M N} \\
=\int d^{4} x \sqrt{-g}\left(e^{2 \phi} \widehat{T}_{\mu \nu} g^{\mu \nu}+e^{2(\Theta+\phi)} \widehat{T}_{m n} \widehat{g}^{m n}\right),
\end{aligned}
$$

where we can observe that the four-dimensional stressenergy tensor in the Einstein frame is defined as we said in expression (7).

\section{Competing Interests}

The authors declare that they have no competing interests.

\section{Acknowledgments}

This work was partially supported by CONACYT 167335 , 179881 Grants and PROMEP Grants UGTO-CA-3 and DAIPUG 640/2015. This work is part of the collaboration within the Instituto Avanzado de Cosmología and Red PROMEP: Gravitation and Mathematical Physics under project Quantum Aspects of Gravity in Cosmological Models, Phenomenology and Geometry of Spacetime. One of authors (L. Toledo Sesma) was supported by a Ph.D. scholarship in the graduate program by CONACYT.

\section{References}

[1] P. A. R. Ade, N. Aghanim, M. Arnaud et al., "Planck 2015 results. XVIII. Background geometry and topology," http:// arxiv.org/abs/1502.01593.

[2] E. Russell, C. B. Kilinç, and O. K. Pashaev, "Bianchi I model: an alternative way to model the present-day Universe," Monthly Notices of the Royal Astronomical Society, vol. 442, no. 3, pp. 2331-2341, 2014.

[3] T. Damour and A. M. Polyakov, "The string dilaton and a least coupling principle," Nuclear Physics B, vol. 423, no. 2-3, pp. 532$558,1994$.

[4] J. H. Horne and G. W. Moore, "Chaotic coupling constants," Nuclear Physics B, vol. 432, no. 1-2, pp. 109-126, 1994.

[5] T. Banks, M. Berkooz, S. H. Shenker, G. W. Moore, and P. J. Steinhardt, "Modular cosmology," Physical Review D, vol. 52, no. 6, pp. 3548-3562, 1995.

[6] T. Damour, M. Henneaux, B. Julia, and H. Nicolai, "Hyperbolic Kac-Moody algebras and chaos in KALuza-KLEin models," Physics Letters. B, vol. 509, no. 3-4, pp. 323-330, 2001.

[7] T. Banks, W. Fischler, and L. Motl, "Dualities versus singularities," Journal of High Energy Physics, vol. 1999, no. 1, article 019, 1999.

[8] R. Kallosh, L. Kofman, and A. Linde, "Pyrotechnic universe," Physical Review D, vol. 64, no. 12, Article ID 123523, 18 pages, 2001.

[9] R. Kallosh, L. Kofman, A. Linde, and A. Tseytlin, "BPS branes in cosmology," Physical Review D, vol. 64, no. 12, Article ID 123524, 2001.

[10] D. Baumann and L. McAllister, Inflation and String Theory, Cambridge Monographs on Mathematical Physics, Cambridge University Press, New York, NY, USA, 2015.

[11] J. Khoury, B. A. Ovrut, N. Seiberg, P. J. Steinhardt, and N. Turok, "From big crunch to big bang," Physical Review D, vol. 65, no. 8, Article ID 086007, 2002.

[12] C. Martínez-Prieto, O. Obregón, and J. Socorro, "Classical and quantum time dependent solutions in string theory," International Journal of Modern Physics A, vol. 19, no. 32, pp. 5651-5661, 2004.

[13] J. Khoury, B. A. Ovrut, P. J. Steinhardt, and N. Turok, "Ekpyrotic universe: colliding branes and the origin of the hot big bang," Physical Review D, vol. 64, no. 12, Article ID 123522, 24 pages, 2001.

[14] K. Enqvist, E. Keski-Vakkuri, and S. Rasanen, "Hubble law and brane matter after ekpyrosis," Nuclear Physics B, vol. 64, p. 388, 2001.

[15] G. Veneziano, "Scale factor duality for classical and quantum strings," Physics Letters. B, vol. 265, no. 3-4, pp. 287-294, 1991.

[16] M. Gasperini and G. Veneziano, "Pre-big-bang in string cosmology," Astroparticle Physics, vol. 1, no. 3, pp. 317-339, 1993.

[17] C. J. Isham, "Canonical quantum gravity and the problem of time," in Integrable Systems, Quantum Groups, and Quantum Field Theories, L. A. Ibort and M. A. Rodríguez, Eds., vol. 409 of NATO ASI Series, pp. 157-287, 1993.

[18] K. V. Kuchar, "Time and interpretations of quantum gravity," International Journal of Modern Physics D, vol. 20, no. 3, pp. 386, 2011.

[19] A. P. Billyard, A. A. Coley, and J. E. Lidsey, "Qualitative analysis of string cosmologies," Physical Review D, vol. 59, no. 12, Article ID 123505, 7 pages, 1999. 
[20] A. P. Billyard, A. A. Coley, and J. E. Lidsey, "Qualitative analysis of early universe cosmologies," Journal of Mathematical Physics, vol. 40, no. 10, pp. 5092-5105, 1999.

[21] A. P. Billyard, A. A. Coley, J. E. Lidsey, and U. S. Nilsson, "Dynamics of M-theory cosmology," Physical Review D, vol. 61, no. 4, Article ID 043504, 2000.

[22] M. Cavaglia and P. V. Moniz, "Canonical and quantum FRW cosmological solutions in M-theory," Classical and Quantum Gravity, vol. 18, no. 1, pp. 95-120, 2001.

[23] M. Cavaglia and P. V. Moniz, "FRW cosmological solutions in M theory," in Recent Developments in Theoretical and Experimental General Relativity, Gravitation and Relativistic Field Theories. Proceedings, 9th Marcel Grossmann Meeting, MG'9, Rome, Italy, July 2-8, 2000. Pts. A-C, 2000.

[24] C. Brans and R. H. Dicke, "Mach's principle and a relativistic theory of gravitation," Aps Journals Archive, vol. 124, no. 3, p. 925, 1961.

[25] D. H. Wesley, "Oxidised cosmic acceleration," Journal of Cosmology and Astroparticle Physics, vol. 2009, article 041, 2009.

[26] J. A. Casas, J. Garcia-Bellido, and M. Quiros, "On the gravity theories and cosmology from strings," Nuclear Physics B, vol. 361, no. 3, pp. 713-728, 1991.

[27] E. J. Copeland, A. Lahiri, and D. Wands, "Low energy effective string cosmology," Physical Review D, vol. 50, no. 8, pp. 48684880, 1994.

[28] J. E. Lidsey, D. Wands, and E. J. Copeland, "Superstring cosmology," Physics Reports, vol. 337, no. 4-5, pp. 343-492, 2000.

[29] M. Gasperini, Elements of String Cosmology, Cambridge University Press, New York, NY, USA, 2007.

[30] S. M. M. Rasouli, M. Farhoudi, and P. V. Moniz, "Modified Brans-Dicke theory in arbitrary dimensions," Classical and Quantum Gravity, vol. 31, no. 11, Article ID 115002, 26 pages, 2014.

[31] M. P. Ryan and L. C. Shepley, Homogeneous Relativistic Cosmologies, Princeton University Press, Princeton, NJ, USA, 1975.

[32] J. Socorro, "Classical solutions from quantum regime for barotropic FRW model," International Journal of Theoretical Physics, vol. 42, no. 9, pp. 2087-2096, 2003.

[33] B. D. Chowdhury and S. D. Mathur, "Fractional brane state in the early Universe," Classical and Quantum Gravity, vol. 24, no. 10, pp. 2689-2720, 2007.

[34] S. Nojiri, O. Obregón, S. D. Odintsov, and K. E. Osetrin, "Induced wormholes due to quantum effects of spherically reduced matter in large $N$ approximation," Physics Letters B, vol. 449, no. 3-4, pp. 173-179, 1999.

[35] S. Nojiri, O. Obregón, S. D. Odintsov, and K. E. Osetrin, “(Non) singular Kantowski-Sachs universe from quantum spherically reduced matter," Physical Review D, vol. 60, no. 2, Article ID 024008, 1999.

[36] S. Nojiri, O. Obregon, S. D. Odintsov, and K. Osetrin, "(Non)singular Kantowski-Sachs universe from quantum spherically reduced matter," Physical Review D, vol. 60, no. 2, Article ID 024008, 1999.

[37] E. Martinez-Gonzalez and J. L. Sanz, " $\Delta \mathrm{T} / \mathrm{T}$ and the isotropy of the Universe," Astronomy and Astrophysics, vol. 300, p. 346, 1995.

[38] J. Socorro, L. O. Pimentel, and A. Espinoza-García, "Classical Bianchi type I cosmology in K-essence theory," Advances in High Energy Physics, vol. 2014, Article ID 805164, 11 pages, 2014.

[39] G. W. Gibbons and L. P. Grishchuk, "What is a typical wave function for the universe?” Nuclear Physics B, vol. 313, no. 3, pp. 736-748, 1989.
[40] S. Gottlber, L. Z. Fang, and R. Ruffini, Eds., Quantum Cosmology, vol. 3 of Advanced Series in Astrophysics and Cosmology, World Scientific, Singapore, 1987.

[41] J. B. Hartle and S. W. Hawking, "Wave function of the universe," Physical Review D, vol. 28, no. 12, pp. 2960-2975, 1983.

[42] J. H. Bae, "Mixmaster revisited: wormhole solutions to the Bianchi IX Wheeler-DeWitt equation using the Euclideansignature semi-classical method," Classical and Quantum Gravity, vol. 32, no. 7, Article ID 075006, 15 pages, 2015.

[43] P. V. Moniz, Quantum Cosmology: The Supersymmetric Perspective: Fundamentals, vol. 1, Springer, 2010.

[44] V. F. Zaitsev and A. D. Polyanin, Handbook of Exact Solutions for Ordinary Differential Equations, Taylor \& Francis, 2002.

[45] C. Kiefer, "Wave packets in quantum cosmology and the cosmological constant," Nuclear Physics B, vol. 341, no. 1, pp. 273-293, 1990.

[46] C. Kiefer, "Wave packets in minisuperspace," Physical Review D. Particles and Fields, vol. 38, no. 6, pp. 1761-1772, 1988.

[47] K. Bamba, J. de Haro, and S. D. Odintsov, "Future singularities and teleparallelism in loop quantum cosmology," Journal of Cosmology and Astroparticle Physics, vol. 2013, article 008, 2013.

[48] S. Nojiri, S. D. Odintsov, and S. Tsujikawa, "Properties of singularities in the (phantom) dark energy universe," Physical Review D, vol. 71, no. 6, Article ID 063004, 2005.

[49] L. Fernandez-Jambrina, “ $\omega$-cosmological singularities," Physical Review D, vol. 82, no. 12, Article ID 124004, 2010.

[50] J. Socorro, M. Sabido, M. A. Sánchez, and M. G. Frías Palos, "Anisotropic cosmology in Sáez-Ballester theory: classical and quantum solutions," Revista Mexicana de Física, vol. 56, no. 2, pp. 166-171, 2010.

[51] J. Socorro and L. Toledo Sesma, "Time-dependent toroidal compactification proposals and the Bianchi type II model: classical and quantum solutions," The European Physical Journal Plus, vol. 131, no. 3, article 71, 10 pages, 2016. 

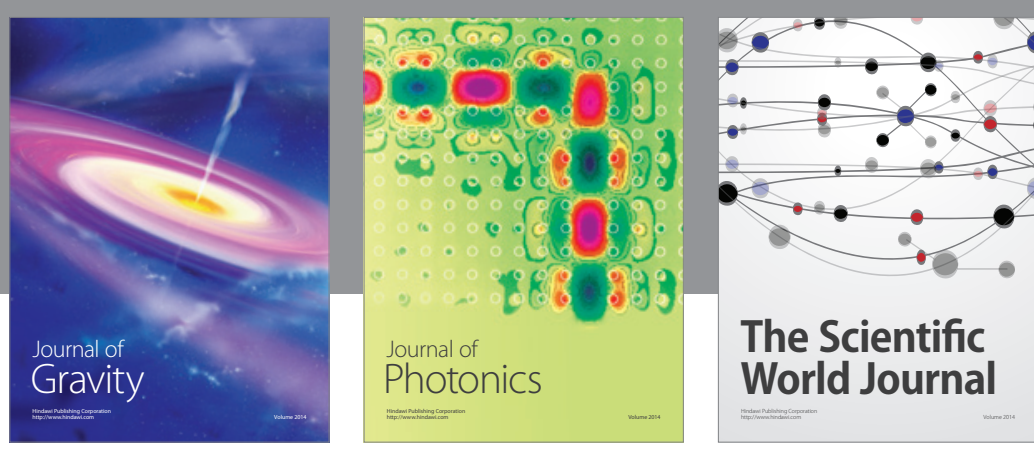

The Scientific World Journal
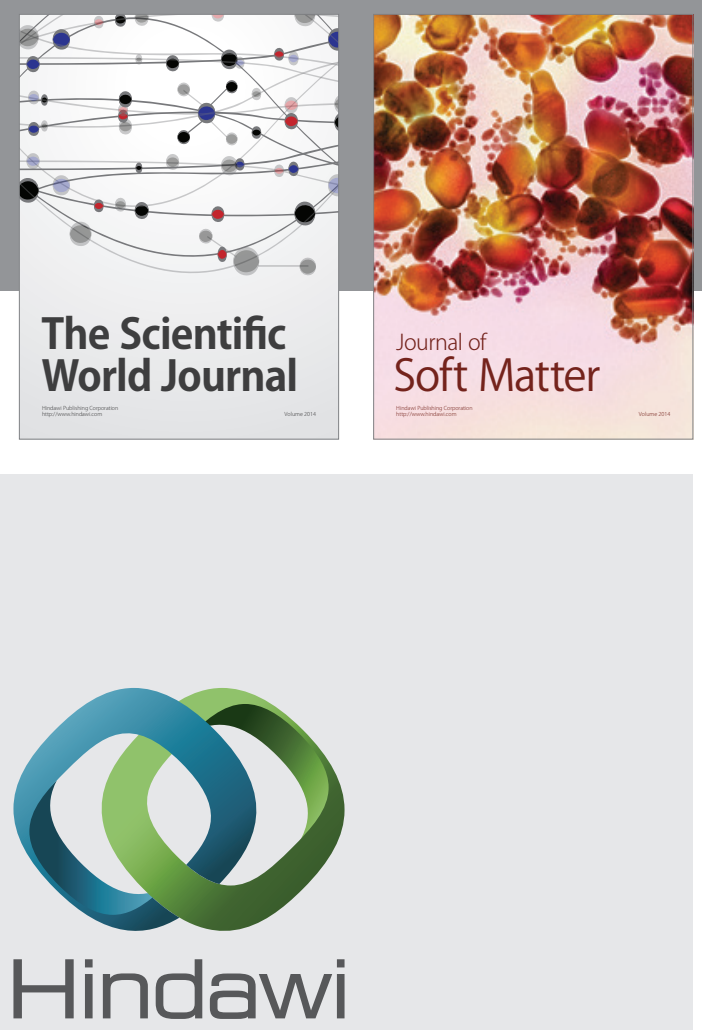

Submit your manuscripts at

http://www.hindawi.com

nternational Journal of

Statistical Mechanics
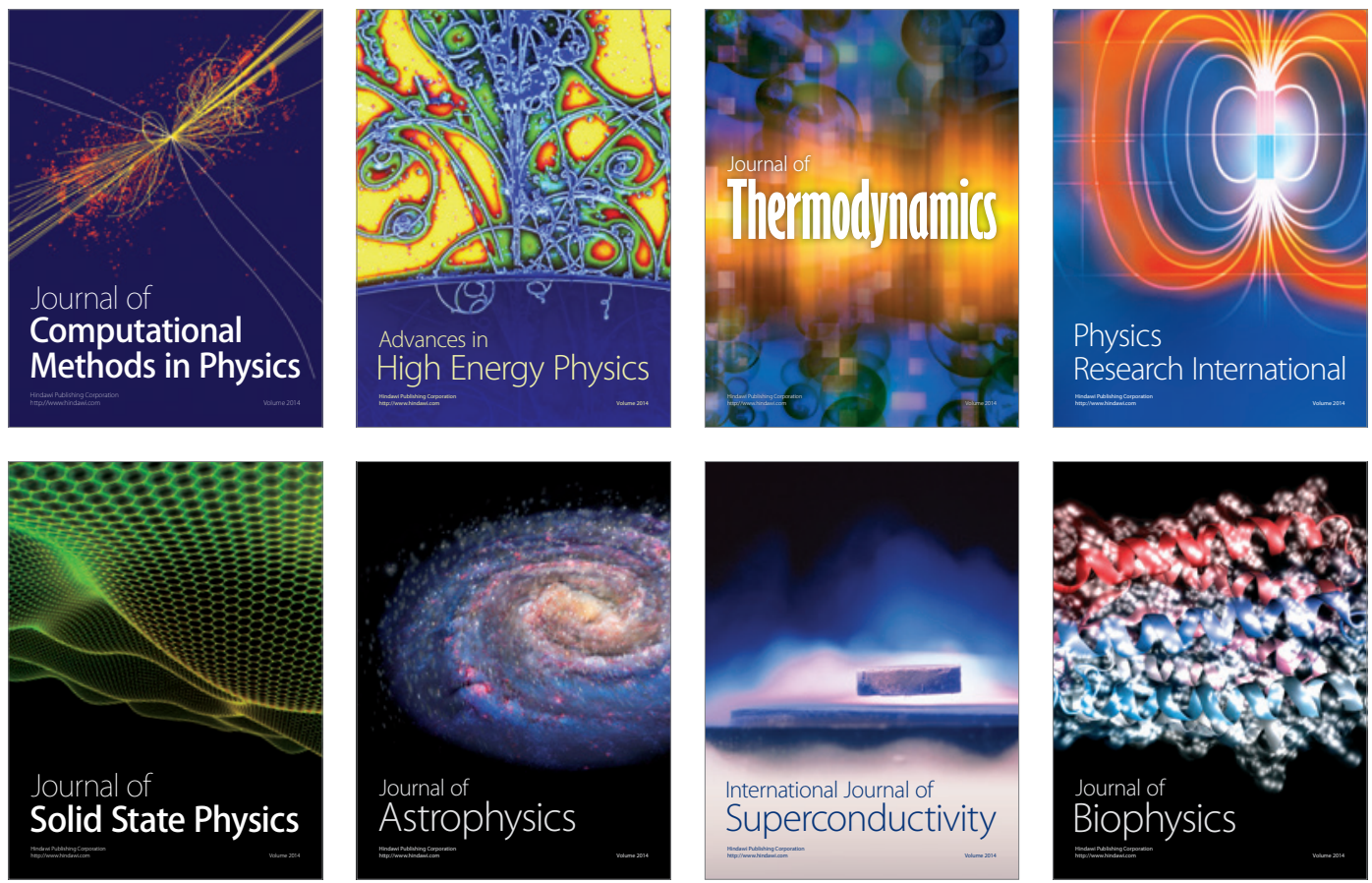
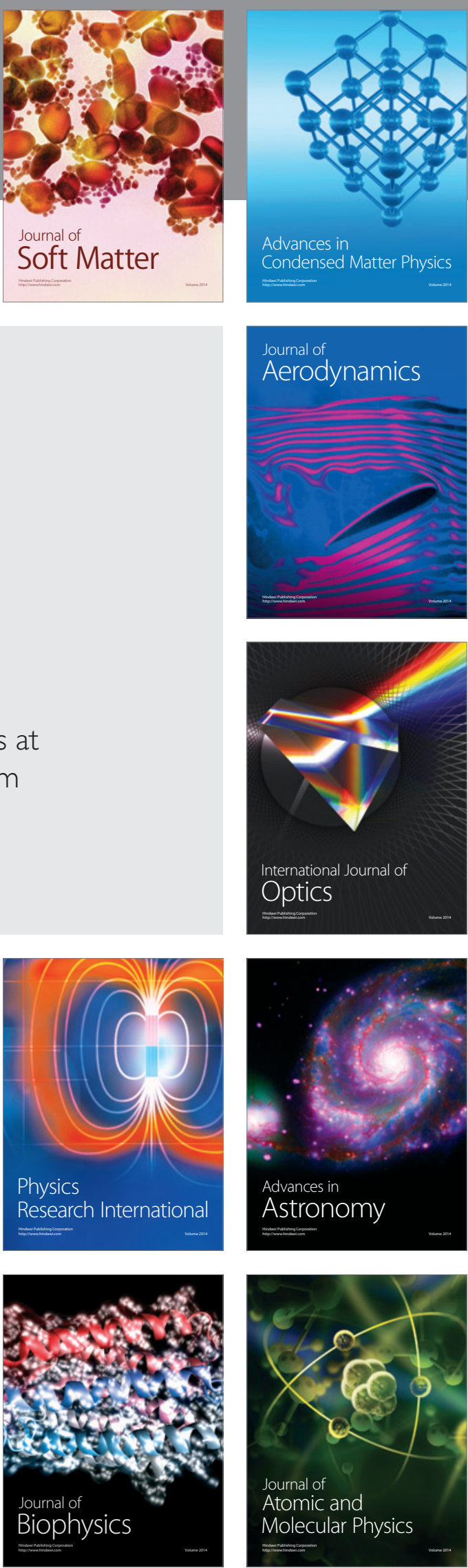